\title{
Foraging Behavior and Patch Time Allocation by Fopius arisanus (Hymenoptera: Braconidae), an Egg-Larval Parasitoid of Tephritid Fruit Flies
}

\author{
Xin-geng Wang ${ }^{1,2}$ and Russell H. Messing ${ }^{1}$
}

Accepted May 28, 2003; revised June 11, 2003

This study quantitatively describes the host-searching behavior of Fopius arisanus (Sonan) (Hymenoptera: Braconidae), an important egg-larval parasitoid of tephritid fruit fly pests, on coffee berries infested with host eggs of the Mediterranean fruit fly, Ceratitis capitata (Wiedemann) (Diptera: Tephritidae). We also investigate the parasitoid's response to local variation in host patch quality. The temporal pattern of behavioral organisation was examined by constructing an ethogram. The parasitoid spent over $90 \%$ of its foraging time in detecting and locating hosts after arriving on a host-infested fruit, and displayed a relatively fixed behavioral pattern leading to oviposition. Patch residence time increased in the presence of host-associated cues, following successful ovipositions, and with increasing size of host clutches per fruit, but decreased with each successive visit to the same host patch and with increasing availability of alternative host patches. The parasitoid females discriminated against previously parasitized hosts and spent significantly less time and searching effort on patches previously exploited by herself or by conspecific females. The effective host-searching behavior, perfect host discrimination ability, and success-motivated searching strategy shown by F. arisanus ensured a thorough exploitation of host resources by this parasitoid.

KEY WORDS: Ceratitis capitata; Fopius arisanus; foraging behavior; parasitoids; patch time allocation.

\footnotetext{
${ }^{1}$ University of Hawaii, College of Tropical Agriculture and Human Resources, 7370 Kuamoo Road, Kapaa, Hawaii 96746.

${ }^{2}$ To whom correspondence should be addressed. Fax: (808) 822 2190. e-mail: xingeng@hawaii. edu.
} 


\section{INTRODUCTION}

Foraging behavior of a parasitoid is directly related to its capacity to locate and parasitize hosts under various conditions and, thus, is a major determinant of its effectiveness for biological control of arthropod pests (Luck, 1990; Wang and Keller, 2002). Also, because of the direct link between successful searching and the production of offspring, parasitoid foraging behavior is likely to be strongly influenced by natural selection and thus has been an ideal subject for testing optimality hypotheses in the context of parasitoid behavioral ecology (van Alphen and Vet, 1986; Godfray, 1994).

One active issue in parasitoid foraging theory is the question of how individual parasitoids optimally allocate their foraging time to patches of different profitability to maximize their lifetime reproduction (Godfray, 1994). An effective parasitoid should concentrate search in areas of high quality host patches to maximize its rate of host attack (Waage, 1983; Wang and Keller, 2002). The present study documents the foraging behavior of Fopius (=Biosteres) arisanus (Sonan), a solitary endoparasitoid attacking eggs of tephritid fruit flies, and describes the effect of host patch quality on the parasitoid's patch residence time.

Fopius arisanus attacks eggs of many tephritid fly pests on a wide variety of fruits and vegetables, and emerges as adults from host puparia (Wharton and Gilstrap, 1983). The parasitoid is native to Southeast Asia and has been introduced into many tropical regions in the world (Wharton, 1989). Since it was introduced into Hawaii in the late 1950s, the parasitoid has outnumbered all larval parasitoids in Hawaii in part because of its competitive superiority (van den Bosch and Haramoto, 1953; Bess et al., 1961; Haramoto and Bess, 1969; Wong et al., 1984; Wong and Ramadan, 1987; Vergas et al., 1993; Purcell et al., 1998; Wang and Messing, 2002; 2003). Currently, F. arisanus is the only egg parasitoid of tephritids in the Western Hemisphere (Wharton and Gilstrap, 1983), and it is considered the most important biological control agent of fruit fly pests (Purcell, 1998).

The developmental and reproductive biology of $F$. arisanus has been well documented (Ramadan et al., 1992, 1994; Bautista et al., 1998). Some aspects of host habitat location by this parasitoid are also known. Vargas et al. (1991) reported that physical appearance of host fruits is an important cue for female F. arisanus host habitat location, with light colors (yellow and white) more attractive than other colors. Other possible cues important to parasitoid attraction are chemical stimuli emitted by ripening fruit (Liquido, 1991; Purcell et al., 1994) and the presence of host fly females on fruits (Haramoto, 1953). Host fruit species, size, shape, and ripeness all have been shown to influence parasitism by F. arisanus (Vargas et al., 1991; Harris and Bautista, 1996). In addition, learning of microhabitats associated with 
host presence can also influence host fruit species preference (Dukas and Duan, 2000). However, detailed knowledge of the foraging behavior and thus foraging efficiency of $F$. arisanus is lacking, despite the unique attributes that make the parasitoid an effective biological control agent of fruit fly pests. In order to predict levels of field parasitism, a more thorough understanding of the parasitoid's behavior is needed.

In this study, we first quantitatively describe the foraging behavior of F. arisanus to show how the parasitoid organized and expressed its hostsearching behavior on coffee berries (Coffea arabica L.) infested with host eggs of the Mediterranean fruit fly, Ceratitis capitata (Wiedemann). We then describe how the parasitoid responded to local variation in host patch quality. These two characteristics will improve our understanding of its foraging efficiency.

\section{MATERIALS AND METHODS}

\section{Parasitoid, Hosts, and Host Fruit}

Fopius arisanus and C. capitata were provided weekly by the USDAARS Pacific Basin Agricultural Research Center, Honolulu, Hawaii, where the parasitoid was reared according to methods described by Bautista et al. (1999). Parasitoid colonies were maintained in wood and screen cages $(25 \times 25 \times 25 \mathrm{~cm})$ under laboratory conditions $\left(22 \pm 2^{\circ} \mathrm{C}, 65 \pm 10 \% \mathrm{RH}\right.$, 12L:12 D), with a 3500-lux fluorescent cool white light and natural light through windows during photophase. Water and honey were provided for the wasps upon their emergence. Ceratitis capitata populations were maintained on artificial diet in the same laboratory. Newly formed puparia were collected into cages, and water and food were provided for adult flies.

Fopius arisanus males emerge 1-2 days earlier than females (Wang and Messing, 2003), and mating usually starts several days after emergence of male wasps (Quimio and Walter, 2000). Female wasps emerge with a partial complement of eggs and then mature more eggs quickly, reaching a peak at 4-6 days after emergence (Ramadan et al., 1992). Thus, we used 6- to 7-day-old female wasps that had been housed with males since eclosion and were presumed to have mated and were never exposed to hosts before the experiments (i.e., naïve). One day prior to the experiments, individual female wasps were aspirated from the holding cage and isolated in plastic cups $(3 \times 3 \times 5 \mathrm{~cm})$ with a drop of undiluted honey on the wall of the cup and water absorbed in a piece of tissue paper.

Because host eggs are concealed within fruit, it is difficult but crucial to determine when and how a $F$. arisanus female wasp is laying an egg when she probes her ovipositor inside a host-infested fruit. We first conducted 
preliminary observations to identify a behavior specific to parasitoid oviposition. A single 5-cm-long groove was cut on the surface of a papaya fruit, and the fruit was then exposed to a group of sexually mature $C$. capitata females in a cage. The flies preferred to lay eggs into the groove. A thin slice of fruit containing the fly eggs was then carefully removed and fitted between two glass microscope sides $(5 \times 3 \times 0.7 \mathrm{~cm})$, and a female wasp was released onto the fruit surface. We could view the host eggs and the parasitoid's ovipositor through the glass slides as she probed the fruit under a dissecting microscope. It was observed that the wasp started pushing her ovipositor through the gap between the glass slides. She stopped probing and remained motionless once her ovipositor detected a host egg. The wasp then slightly pierced the host egg's chorion and inserted the tip of her ovipositor inside the host egg. This "motionless" behavior lasted for an average of $134 \mathrm{~s}$ (range, 56-252 s; based on observations of 22 females) from the onset of an examination to ovipositor withdrawal after laying an egg. Actual oviposition was confirmed by dissecting the host egg following each observation. No motionless period was observed to be $>50 \mathrm{~s}$ in the absence of egg laying. Therefore, we used the observed behavior of a motionless body for $>50 \mathrm{~s}$ as a criterion to determine if the parasitoid was laying an egg into a host when she was probing inside a host-infested fruit in later experiments.

Coffee berries, a major host of C. capitata, were used as host fruit in this study. One day before experiments, coffee twigs harboring mature fruit were cut from a coffee orchard at the Kauai Agricultural Research Center. All the fruit were carefully examined under a stereomicroscope in the laboratory for the presence of oviposition scars by fruit flies and were rinsed thoroughly in tap water to remove host-associated residue. Only mature, uninfested, and intact fruits were selected for experiments. All coffee twigs were cut into 5-cm lengths, each harboring one, two, or three fruit (all others were removed), and were individually inserted into $200-\mathrm{ml}$ vials filled with water. Each coffee twig was considered as one experimental patch.

\section{Foraging Behavior}

We conducted observations on the foraging behavior of $F$. arisanus in a Plexiglas and screen cage $(30 \times 30 \times 30 \mathrm{~cm})$ under the laboratory conditions previously described. A single-fruit coffee twig was used as an experimental patch. One day prior to the experiment, the twig was exposed to a group of 10-day-old female flies of $C$. capitata in a cage. When one fly was observed to start boring the fruit surface with her ovipositor, all other flies searching or sequentially landing on the fruit were brushed away until the ovipositing fly withdrew her ovipositor and marked the fruit surface (after laying a clutch 
of eggs) with typical ovipositor-dragging behavior (see Papaj et al., 1989). The twig was then removed from the cage. Therefore, the fruit received only a single clutch of host eggs. On average it took $216 \pm 11.8 \mathrm{~s}(n=30)$ for a fly to lay a single clutch of eggs in a coffee fruit, and final dissections after the experiments showed that each clutch contained $3.7 \pm 0.25$ eggs (range, 2-7 eggs). The flies laid eggs either into the thin space between the two coffee seeds, into the ends of the fruit, or between the seeds and the fruit surface. It was noted that some of the eggs were inserted deeper, while others were near the surface of a puncture cavity.

The experimental patch (twig) was then placed in the middle of a test cage. An additional patch consisting of a single uninfested coffee fruit was placed $5 \mathrm{~cm}$ away from the experimental patch to provide an alternative site for the wasp to land when she decided to leave the experimental patch. Otherwise, a wasp might spend excessive amounts of time on an experimental patch if there were no alternative sources of semiochemicals (Wang and Keller, 2002). A single female wasp was then released directly onto the experimental patch by holding the wasp in a small plastic cup $(3 \times 3 \times 5 \mathrm{~cm})$ and allowing her to walk onto the fruit. Immediately before the release, the wasp was caged inside a plastic cup for 5 min with a fresh coffee fruit containing a needle puncture to stimulate her searching behavior. This exposure increased the wasp's direction towards the experimental patch once released. If the wasp did not successfully walk onto the fruit or did not immediately start searching once arriving onto the fruit, it was discarded.

Once the wasp started searching on the fruit, its behavior was continuously recorded using a event-recording software (The Observer for Windows 3.0, Noldus Information Technology, The Netherlands). The time was recorded to the nearest $1 \mathrm{~s}$. The following eight distinctive behavioral events were defined based on preliminary observations and were recorded during the observations.

(1) Antennating. The wasp holds its antennae close to each other and palpates the fruit surface with both antennal tips while either moving or stationary. Usually the wasp moves rather slowly and in varying directions.

(2) Probing. The wasp stops antennating and raises her antennae over her head, spreading them about $40-50^{\circ}$ from each other. She then raises her abdomen and bends her ovipositor and drills the ovipositor inside the fruit. Often the wasp turns around anticlockwise and clockwise and pushes her ovipositor back and forth while drilling.

(3) Detecting. The wasp sometimes pierces the fruit surface using her ovipositor while antennating. This behavior is distinct from the probing behavior, in which antennae are raised. 
(4) Ovipositing. The wasp stops pushing or drawing her ovipositor, except that the tip of her ovipositor pierces a host egg surface while the rest of the body stays motionless for a certain period with the antennae raised (see detailed description above). During the observations, all observed periods of "motionless" behavior were first recorded as "oviposition." After the observation, only those with a duration of $>50 \mathrm{~s}$ were counted as ovipositions in later data analysis, while the rest of the records were treated as a part of probing behavior. Later dissections indicated that the actual number of ovipositions matched well with the observed frequency of the motionless behavior.

(5) Grooming. The wasp repeatedly brushes its ovipositor with its hindlegs, rubs legs together, and does other actions involving cleaning its body.

(6) Resting. The wasp keeps motionless on the fruit surface with its antennae and ovipositor stretched out.

(7) Walking. The wasp moves along the fruit surface at a relatively constant speed with the antennae extended in front of the head.

(8) Flying. The wasp stops walking or antennating, points her body to the air, and then flies away.

An observation was terminated when the wasp flew away from the fruit or walked off the experimental patch. A total of 25 complete observations was collected. Each fruit and wasp was used only once. Following each observation, all host eggs in the fruit were removed and individually dissected to determine the presence of parasitoid eggs, and each experimental wasp was immediately dissected to determine her mature egg load.

The mean duration, number of occurrences, and proportion of time devoted to each defined behavior were calculated for each individual wasp. The temporal pattern of behavioral flow was then examined by constructing an ethogram. The goal of this analysis was to gain an insight into how behavior was organized, rather than to develop a robust model of behavior. First-order Markovian behavioral transition matrices were constructed for each individual, with rows corresponding to preceding behaviors and columns to following behaviors (Goodman, 1968). Data from all 25 individuals were pooled in the analysis. By pooling we assume that there were no significant differences in behavioral flow among individuals. The expected values of the matrix cells were found using the iterative proportional fitting method of Goodman (1968), and the statistical significance of the overall table was evaluated using a log-likelihood ratio $(G)$ test, with Yates' correction for continuity applied throughout. When the overall table test was found to be statistically significant, significant transitions were found 
by collapsing the table into a $2 \times 2$ matrix around each transition and a $G$ test was performed. The significance of these individual tests was adjusted to a tablewide level of $5 \%$ using the sequential Bonferroni method (Rice, 1989). We used a computer program developed by M. A. Keller (Field and Keller, 1993) to analyze the behavioral transitions, with the results presented graphically in an ethogram (Field and Keller, 1993; Wang and Keller, 2002).

\section{Patch Residence Time}

Four experiments were conducted to investigate the possible effects of various factors on patch residence time by $F$. arisanus. As described above, each experiment was carried out in a Plexiglas and screen cage $(30 \times$ $30 \times 30 \mathrm{~cm})$ in the laboratory.

Effect of Presence of Host-Associated Cues and Ovipositions. A singlefruit coffee twig was used as an experimental patch. The experiment consisted of four fruit treatments: (a) clean fruit without prior exposure to host flies; (b) fruit contaminated by allowing the host flies to search over the surface only; (c) fruit containing one host oviposition puncture only and no eggs; and (d) fruit infested with a single clutch of host eggs. For the second and third treatments, the fruit was exposed to a group of 10 flies. We allowed the flies to search over the fruit surface but any attempt of ovipositor boring was stopped with a fine brush (treatment $b$ ) or we allowed one fly to bore only one puncture but then the fly was brushed away before she laid any eggs (treatment c). To obtain an infestation with a single clutch of $C$. capitata eggs, the same procedure described in the behavioral observation experiments above was followed. The four treatments were replicated 20, 20, 27, and 29 times, respectively.

Effect of Host Egg Density per Clutch (or Cluster). As in Experiment 1, a single-fruit coffee twig was used as an experimental patch, and the fruit was exposed to a group of 10 flies to allow a female fly to lay only one clutch of eggs (as in the last treatment of the above experiment). In addition, here we allowed another one to three female flies to subsequently lay eggs into the same puncture that the first female made so that we could obtain a varying density of host eggs per cluster. A total of 69 patches of host fruits was collected and tested. Final dissections after the experiments showed that the host egg density varied from 2 to 25 eggs per cluster.

Effect of Previous Parasitism. A two-fruit coffee twig was used as an experimental patch. In order to obtain infestation of each fruit with a single clutch of host eggs, we first wrapped one fruit with Parafilm and exposed the other fruit to host flies following the same procedure as described in the 
behavioral observation experiments above. After oviposition was observed, the infested fruit was wrapped with fresh Parafilm, the Parafilm on the uninfested fruit was immediately removed and the uninfested fruit was exposed to the flies until it also received a single clutch of host eggs.

Following the infestation of the fruits by the flies, one of the two fruits was randomly selected and wrapped with Parafilm while the other one was exposed to a female wasp to allow her to parasitize the host eggs until she freely left the fruit. The wasp was then recovered from the fruit and caged into a plastic cup with honey and water, and the Parafilm was removed from the fruit. Each time at least two experimental patches were prepared using two different female wasps. On the following day, the wasp experienced with oviposition in one patch was released onto the other patch in which one of the fruits was exploited by a conspecific female on the previous day. Wasps were released either on the exploited or unexploited fruit first in random order. The experiment was repeated 10 times for each release order (20 replicates total).

Effect of Size of the Host Patch. In nature, a coffee twig harbors several to dozens of fruits. This experiment was designed to investigate the effect of the availability of nearby host-infested fruits on the parasitoid's residence time on one fruit. An experimental patch consisted of one (same as the last treatment of Experiment 1), two, or three fruit, with each fruit infested with a single clutch of host eggs within a 2-h period following the same procedures as described in Experiment 2. Individual naïve wasps were then released on one of the randomly selected fruits. The three treatments were repeated 29 , 36, and 24 times, respectively.

For all of the above experiments, an extra patch consisting of a single uninfested coffee fruit was placed $5 \mathrm{~cm}$ away from the experimental patch. Patch residence time, number of probes in each host oviposition puncture, and number of ovipositions on each fruit were recorded using the Observer software. For Experiment 4 these parameters were recorded for each fruit. An observation was terminated when the wasp flew away or walked off the experimental patch. Each wasp and experimental patch were used only once. Following each observation, all host eggs in the fruit were removed and individually dissected to determine the presence of parasitoid eggs. In Experiment 3, the size of parasitoid eggs deposited $24 \mathrm{~h}$ prior to the experiments differed substantially from those deposited during the experiments, so it was easy to distinguish the older eggs from recently laid eggs.

Patch residence time, defined as the time from wasp arrival on the experimental patch until she left it, was calculated for each wasp. Giving-up time, defined as the time during which a female wasp remained in the patch following the last oviposition (Waage, 1979), was also calculated for Experiment 1. 
We analyzed the effect of the presence of host-associated cues and successful ovipositions on patch residence time (Waage, 1979; Driessen et al., 1995) and giving-up time using a log-rank test on survival analysis using a Bonferroni procedure for multiple comparison (Kaplan-Meier procedure; JMP 4.0, SAS).

In Experiment 2, the data points representing percentage parasitism often overlapped when they were plotted against host egg density per cluster, particularly when the egg density per cluster was lower than 5 eggs (in most of these cases, the resultant percentage parasitism was 100). The host density per clutch was therefore sorted into three ranges: $2-5$ eggs (about one clutch size), 6-10 eggs (about two clutch size), and 11-25 eggs ( $>$ two clutches) for the data analysis.

All other comparisons between or among treatments were analyzed using Kruskal-Wallis one-way AOV (Statistix for Windows, 4.0).

\section{RESULTS}

\section{Foraging Behavior}

The temporal pattern of behavioral organisation, flow, frequency, duration, and proportion of time devoted to each type of behavior by F. arisanus is summarized in Fig 1. When a female wasp arrived on a host-infested coffee fruit, she immediately palpated the fruit surface with the tips of her antennae. At times, the wasp also probed the fruit surface with her ovipositor while antennating. These behaviors were associated with intensive searching activity in the presence of host-associated cues. Once her antennae encountered a host oviposition puncture, the wasp stopped antennating, directly approached the site, raised its abdomen, and inserted its ovipositor into the puncture. At such sites, the wasp showed a strong arrestment response and often turned around while drilling its ovipositor from different directions, angles, and depths in the fruit to locate a host.

Searching females repeatedly displayed antennating-detecting behaviors before locating a potential host oviposition site. After the location of a clutch of host eggs, she exhibited a stereotyped sequence of behavior (probingovipositing-probing) before she gave up the host oviposition site. She often rested for a while after a long bout of antennating. When foraging on a hostinfested fruit, the wasps spent very little time walking. However, the wasp walked to a different site before leaving and then pointed to the air before taking off. There was no flight when the wasp searched within a fruit patch.

The parasitoid often searched a whole fruit and repeatedly visited and probed a previously discovered host oviposition site before she finally left 


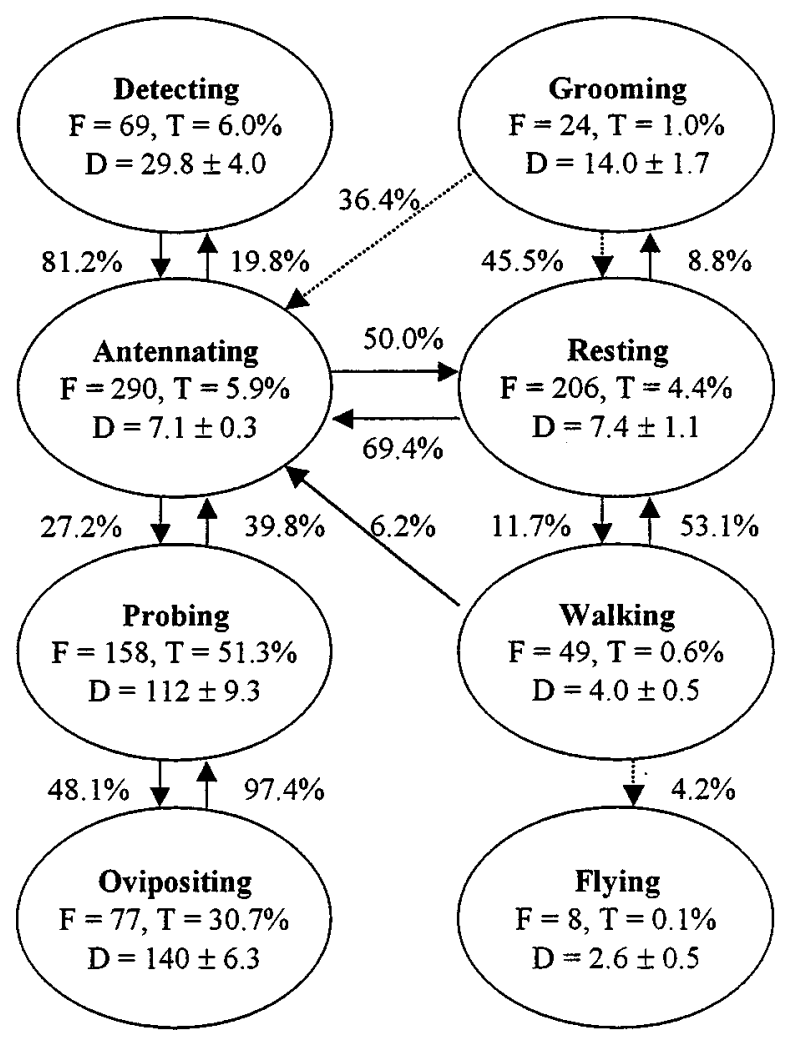

Fig. 1. Ethogram of Fopius arisanus searching on a coffee fruit infested with a single clutch of host eggs of Ceratitis capitata. A solid-line arrow represents a significant behavioral flow $(P<0.05)$, while a dotted-line arrow represents an insignificant behavioral flow $(P>0.05)$. The figures adjacent to the arrows represent the percentage of transitions from given behavioral events (transitions with a frequency lower then $5 \%$ are not shown). F, total frequency of each behavioral event; T, percentage of time devoted to each behavioral event; $\mathrm{D}$, average $( \pm \mathrm{SE})$ duration (s) of a single behavioral act $(n=25$ wasp observations). All observed flying behaviors followed walking and the overall frequency of flying was too low to perform a statistical test of significance.

the fruit. Overall, the parasitoid spent the greatest proportion of its time in locating and parasitizing hosts (probing, 51.3\%; ovipositing, 30.7\%). Its foraging behavior was dominated by a high frequency of antennating and long periods of probing and ovipositing with short and frequent resting periods. 
Grooming was less frequently observed in the parasitoids. The wasps left the experimental patches by walking off (17 cases) or flying away (8 cases).

On average, each $F$. arisanus female $(n=25)$ laid $3.0 \pm 0.25$ eggs before she left the experimental patch. In 17 of 25 cases the wasp parasitized all the host eggs before it left the experimental patch. Not a single incident of selfsuperparasitism was found. The mean mature egg load of the wasps $(n=25)$ as determined by dissection was $89.3 \pm 5.0$ after the experiments.

\section{Patch Residence Time}

The presence of host-associated cues, host oviposition punctures, and successful ovipositions significantly increased the parasitoid's residence time on the experimental patch (Table I). The parasitoid quickly left when she landed on an uninfested host patch. In 6 of 20 cases, the wasps directly walked off the host fruits without antennal searching. There was no significant difference in residence time by the parasitoid between patches contaminated by host-associated cues and patches that contained a host oviposition puncture without eggs (Table I). On host egg-infested patches the giving-up time $(694 \pm 67.5 \mathrm{~s} ; n=29)$ after successful ovipositions was significantly longer than the residence time on patches which had host oviposition punctures only and no host eggs $(255 \pm 46 \mathrm{~s} ; n=27)$ (Kruskal-Wallis tests, $P<0.001)$.

On the patches containing a host-puncture only, the wasps probed a mean of $1.5 \pm 0.14$ times (range, $1-3$ times; $n=27$ ) inside the punctures before they left the host patches. When wasps visited host egg-infested patches $(n=29)$, they probed an average of $3.0 \pm 0.27$ times inside the punctures and laid $2.6 \pm 0.21$ eggs before they left the patch. Every wasp tested laid at least one egg before she gave up the patch. No single occurrence of superparasitism was observed during the entire experiment.

Table I. Patch Residence Time by Fopius arisanus on Coffee Fruits That (a) Were Clean; (b) Were Contaminated Externally by Host-Associated Cues Only; (c) Contained a Single Oviposition Puncture Only, and No Eggs; and (d) Were Infested with a Single Clutch of Host Eggs of Ceratitis capitata

\begin{tabular}{lcc}
\hline \multicolumn{1}{c}{ Treatment of host fruit } & $N$ & Patch residence time (s) \\
\hline Clean & 20 & $17.2 \pm 2.4 \mathrm{a}^{a}$ \\
Contaminated by host-associated cues & 20 & $161 \pm 27 \mathrm{~b}$ \\
Containing a host oviposition puncture & 27 & $255 \pm 46 \mathrm{~b}$ \\
Infested with one clutch of host eggs & 29 & $1054 \pm 106 \mathrm{c}$ \\
\hline
\end{tabular}

${ }^{a}$ Values (mean $\pm \mathrm{SE}$ ) in the same column followed by a different letter are significantly different (log-rank tests of survival analysis, Kaplan-Meier procedure; JMP 4.0, SAS). 
Both patch residence time and giving-up time after successful oviposition on host egg-infested patches were significantly longer than on patches which had oviposition punctures only, and no host eggs (Table I), suggesting that ovipositions by the parasitoid increased the parasitoid's patch residence time.

Both patch residence time and oviposition rate per unit time by the parasitoids increased with increasing clutch size of host eggs in the fruit (Figs. 2A and B). The resultant percentage parasitism was not density dependent within this host egg density range (Fig. 2C). However, the percentage of host eggs that escaped from attacks by the parasitoid increased with increasing host density per clutch (Fig. 2C).

The parasitoid spent more time searching on unexploited fruit than on fruit previously exploited by a conspecific female, regardless of which fruit was visited first, although there was no significant difference in the number of visits to different fruit (Table II). The mean number of ovipositions on the unexploited fruit was significantly greater than on the previously exploited fruit (Table II). No superparasitism by F. arisanus was observed on either fruit. Each tested wasp had experienced at least one oviposition in an unparasitized host on the day prior to the tests. These results suggest that the experienced wasps discriminated against hosts previosly parasitized by a conspecific female, with such discrimination ability probably obtained through examination of a mark left on the surface or inside of the host eggs rather than on the surface of the host fruit (or patch surface). The discrimination resulted in an early leaving of the exploited fruit. However, when the wasps were released onto the exploited fruit first the number of probes was significantly smaller on the exploited patch than on the unexploited patch

Table II. Searching Efforts by Fopius arisanus on Coffee Fruit Previously Unexploited vs. Exploited by a Conspecific Female ( $N=10$ Wasps): Both Fruit Were Initially Infested with One Clutch of Host Eggs of Ceratitis capitata

\begin{tabular}{|c|c|c|c|c|}
\hline \multirow[b]{2}{*}{ Searching effort } & \multicolumn{2}{|c|}{ Visit unexploited fruit first ${ }^{a}$} & \multicolumn{2}{|c|}{ Visit exploited fruit first } \\
\hline & Exploited & Unexploited & Exploited & Unexploited \\
\hline Host egg density per clutch & $4.3 \pm 0.54 \mathrm{a}^{b}$ & $4.3 \pm 0.60 \mathrm{a}$ & $3.6 \pm 0.45 \mathrm{a}$ & $4.3 \pm 0.67 \mathrm{a}$ \\
\hline Hosts previously parasitized & $3.1 \pm 0.55 \mathrm{a}$ & 0 & $2.8 \pm 0.47 \mathrm{a}$ & 0 \\
\hline$\%$ time allocation & $38.2 \pm 3.2 \mathrm{a}$ & $61.8 \pm 3.2 \mathrm{~b}$ & $26.7 \pm 5.1 \mathrm{a}$ & $73.3 \pm 5.1 b$ \\
\hline No. of visits & $2.1 \pm 0.23 \mathrm{a}$ & $1.9 \pm 0.31 \mathrm{a}$ & $2.3 \pm 0.26 \mathrm{a}$ & $2.5 \pm 0.27 \mathrm{a}$ \\
\hline No. of probes & $3.1 \pm 0.41 \mathrm{a}$ & $3.6 \pm 0.65 \mathrm{a}$ & $1.6 \pm 0.40 \mathrm{~b}$ & $3.8 \pm 0.44 \mathrm{a}$ \\
\hline No. of ovipositions & $0.3 \pm 0.21 \mathrm{a}$ & $2.9 \pm 0.75 b$ & $0.6 \pm 0.50 \mathrm{a}$ & $3.3 \pm 0.60 \mathrm{~b}$ \\
\hline
\end{tabular}

${ }^{a}$ Wasps were released either on the exploited or unexploited fruit first.

${ }^{b}$ Mean $( \pm$ SE) values in the same row followed by a different letter are significantly different (Kruskal-Wallis tests, $P<0.05$ ). 

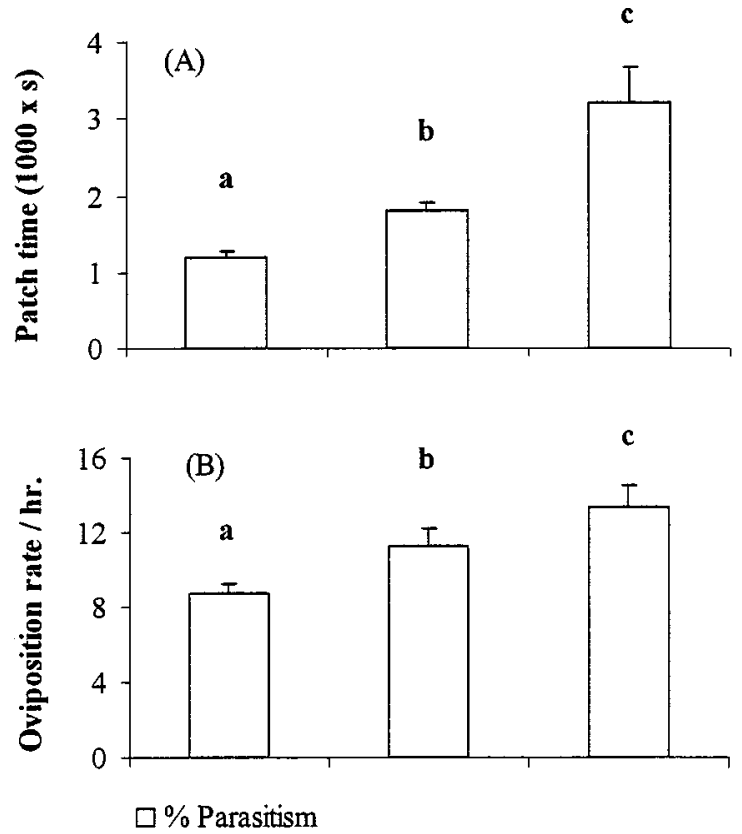

Egg clusters in which all hosts were parasitized

(C)

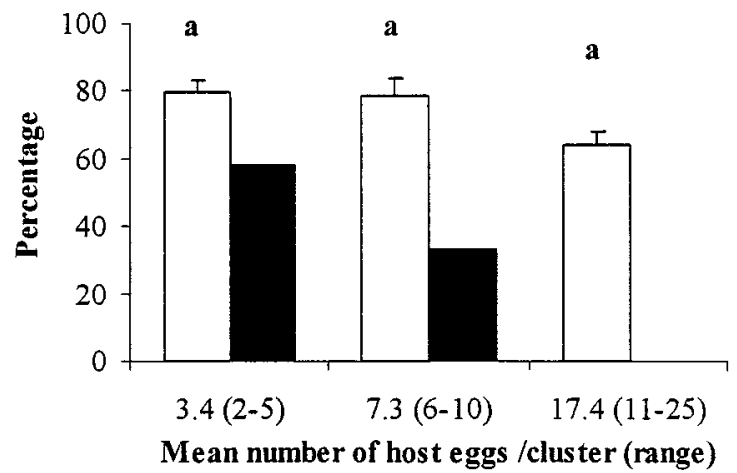

Fig. 2. The relationship between the clutch size of Ceratitis capitata eggs per fruit and (A) patch residence time, (B) oviposition rate per unit time, and $(\mathrm{C})$ the resultant rate of parasitism by Fopius arisanus. The numbers of replicates for the three host egg density ranges per cluster are 29 (2-5), 22 (6$10)$, and 18 (11-25). Error bars represent one standard error, and different letters above each bar indicate significant differences among treatments (Kruskal-Wallis tests, $P<0.05$ ). 
Table III. Patch Residence Time per Fruit Visit, Number of Visits to, and Number of Ovipositions in Each Fruit by Fopius arisanus as Affected by the Size of the Patch That Consisted of a

Varying Number of Fruit, Each Infested by One Clutch of Host Eggs of Ceratitis capitata

\begin{tabular}{lccc}
\hline \multirow{2}{*}{\multicolumn{1}{c}{ Searching effort }} & \multicolumn{3}{c}{ Patch size (number of fruit) } \\
\cline { 2 - 4 } & One & Two & Three \\
\hline$N$ & 29 & 36 & 24 \\
No. of host eggs per clutch & $3.7 \pm 0.22 \mathrm{a}^{a}$ & $4.5 \pm 0.31 \mathrm{a}$ & $3.7 \pm 0.41 \mathrm{a}$ \\
No. of visits per fruit & $1.0 \pm 0.0 \mathrm{a}$ & $1.8 \pm 0.23 \mathrm{~b}$ & $2.9 \pm 0.37 \mathrm{c}$ \\
Residence time per visit (s) & $1054 \pm 106 \mathrm{a}$ & $747 \pm 145 \mathrm{~b}$ & $362 \pm 53 \mathrm{c}$ \\
No. of ovipositions per fruit & $2.6 \pm 0.21 \mathrm{a}$ & $2.1 \pm 0.38 \mathrm{a}$ & $2.0 \pm 0.37 \mathrm{a}$ \\
Oviposition rate during the & $6.4 \pm 0.85 \mathrm{a}$ & $17.7 \pm 4.01 \mathrm{~b}$ & $24.1 \pm 4.7 \mathrm{c}$ \\
$\quad$ & & & \\
\hline first visit (No. of eggs/h) & & & \\
\hline
\end{tabular}

${ }^{a}$ Mean $( \pm \mathrm{SE})$ values in the same row followed by a different letter are significantly different. All tests were compared using Kruskal-Wallis tests $(P<0.05)$, except for patch residence time, in which case log-rank tests of survival analysis (Kaplan-Meier procedure; JMP 4.0; SAS) were performed.

(Table II), suggesting that foraging experience with exploited fruit further influenced the parasitoid's patch residence time.

With increasing availability of alternative host fruits in the immediate environment, the parasitoid stayed a shorter time on each visit to a host-infested fruit but paid more return visits to the same fruit (Table III). The first visit by the parasitoid was far longer than each successive return visit (Fig. 3A) and eggs were laid mainly during their first visit to the fruits (Fig. 3B). Although there was no difference in the mean number of ovipositions per fruit among the different patch sizes, oviposition rate during the first visit increased significantly with increasing number of fruits in the patches (Table III).

\section{DISCUSSION}

Although $F$. arisanus is a generalist parasitoid, our study showed that it displayed a relatively fixed pattern leading to host location (Fig. 1), a behavioral trait similar to those of many efficient host-specific parasitoids (van Alphen and Vet, 1986; Godfray, 1994; Wang and Keller, 2002). F. arisanus locates a potential host oviposition site through antennal search and then locates hosts through ovipositor probing in the host-infested fruit. As host eggs are completely concealed from the foraging parasitoid, the functions of these behaviors are self-evident and similar to other fruit fly parasitoids of this subfamily that search for frugivorous hosts (e.g., Greany et al., 1977; Messing and Jang, 1992). 

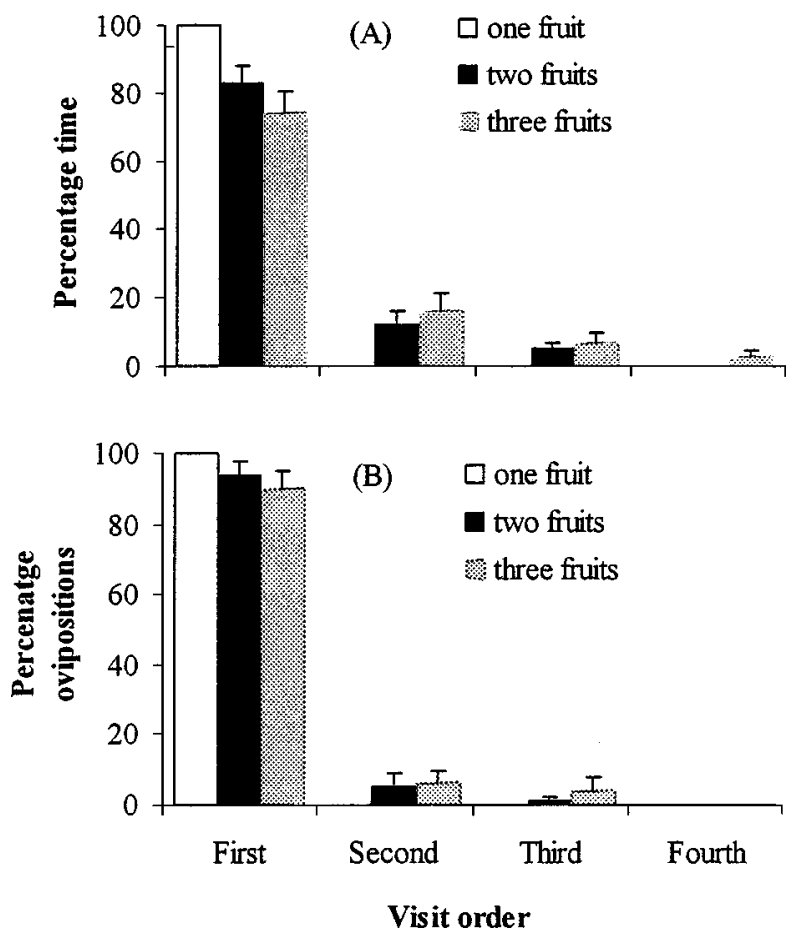

Fig. 3. Percentage $( \pm \mathrm{SE})$ of $(\mathrm{A})$ searching time allocation and (B) ovipositions by Fopius arisanus on each successive visit to the same host fruit in patches consisting of different numbers of host fruit each infested with one clutch of host eggs of Ceratitis capitata. Error bars represent one standard error (Kruskal-Wallis tests, $P<0.05)$.

Fopius arisanus adjusts its patch residence time in response to local variations in host patch quality. Presence of host-associated cues, possibly the odors emitted from a host oviposition scar and kairomon residue of adult flies on the fruit surface, increased the parasitoid's residence time on the host patch. Similar responses to the presence of host-associated cues have been demonstrated in many other parasitoid species (van Alphen and Galis, 1986; Waage, 1979; Nelson and Roitberg, 1995; Wang and Keller, 2002). Kairomone concentrations are assumed to be used by a parasitoid to assess initial patch quality and to establish a basic tendency to stay there upon entering a given patch, for example, in Venturia canescens (Grav.) (Waage, 1979). However, the presence of host kairomones may be only an indication of potential host resources rather than a reliable measure of patch quality, 
as other conspecific females or other parasitoid species may have previously exploited the host patch. Thus, an effective parasitoid should use withinpatch foraging experience to assess actual patch quality and adjust its patch residence time accordingly (Vos et al., 1998; Wang and Keller, 2003).

Waage (1979) proposed a behavioral mechanism model which assumes that a parasitoid should leave a patch after a certain time if nothing else happens, while ovipositions increase (incremental mechanism [Waage, 1979]) or decrease (countdown mechanism [Driessen et al., 1995]) its responsiveness by a set amount that is assumed to be a function of unsuccessful search time since last oviposition (Waage, 1979) or number of ovipositions (Driessen et al., 1995). Many empirical studies have supported either of the above models (see Driessen and Bernstein, 1999). Our results suggest that at least one oviposition by $F$. arisanus significantly increased her residence time on the patches, compared to patches infested with host punctures but no eggs. This agrees with the prediction of Waage's (1979) model that ovipositions increase patch residence time. The adaptive value determining which mechanism a parasitoid should use may depend on the density and distribution of its hosts and a parasitoid's ability to perfectly assess the initial patch quality (van Alphen and Vet, 1986; Godfray, 1994; Driessen et al., 1995). With a perfect assessment of initial patch quality, a parasitoid should use a decremental mechanism, particularly in an environment with low and uniformly distributed host resources, as each oviposition provides information regarding the loss of future value of the patch (Driessen et al., 1995). In contrast, if a parasitoid has incomplete information regarding the initial patch quality, Waage's mechanism should be the more adaptive strategy, particularly in a rich environment with clustered host resources (Driessen et al., 1995).

Other factors such as host discrimination ability may also affect the patch time allocation mechanism. The risk of superparasitism, and thus waste of search effort, would increase with patch exploitation if a parasitoid could not effectively discriminate against parasitized hosts. Early leaving would thus be an adaptive advantage for parasitoids with imperfect host discrimination ability (van Lenteren, 1991; Rosenheim and Mangel, 1994). Our results suggest that $F$. arisanus appears to have almost-perfect host discrimination ability. There would be little or no risk that additional eggs or time would be wasted. As a result, Waage's mechanism or "success-motivated" search would result in a thorough exploitation of host resources by the parasitoid. In contrast, the opposite mechanism would result in a parasitoid that leaves prematurely from a host-rich patch. In this case, the host fly, $C$. capitata, prefers to oviposit into punctures previously made by conspecific females, which results in multiple clutches deposited in a single puncture (Papaj et al., 1989, 1992), and the number of host eggs per cluster varies greatly as observed in this study. 
With exploitation, the encounter rate with healthy hosts would decrease, as more hosts become unsuitable due to previous parasitization. When alternative resource patches are available, an optimally searching parasitoid should leave each patch when the instantaneous resource harvest rate falls to the average rate expected for all patches, as predicted by the marginal value theorem (Charnov, 1976). Our results agree with this prediction; F. arisanus stayed for a shorter time when in the presence of additional host patches in the immediate environment, which resulted in an overall increased attack of hosts per unit time.

The resultant parasitism by $F$. arisanus was locally density independent in the host density range tested in this study (Fig. 2B). With increasing host density per cluster and patch residence time, the wasps probably encountered and examined more previously parasitized hosts without increasing the level of parasitism. Thus, handling time per host could increase with increasing host density per cluster, which could be a factor contributing to the densityindependent pattern of parasitism observed.

The location of host eggs within each fruit puncture probably affected their rate of parasitization by F. arisanus. The wasp usually drilled her ovipositor deeply into the host puncture cavity. Thus, the eggs at the top or surface of the fruit were less likely to be detected, which could also contribute to the large variations in the observed pattern of oviposition rate among different individuals in different patches (Fig. 2B). It is necessary to point out that we allowed one to three fly females to lay additional eggs into the same puncture in order to obtain a varying density of host eggs per cluster. Thus, the amount of host-associated marking cues may be an additional factor influencing this variation.

Increased egg density in a host fruit, particularly small fruits like coffee berries, is associated with a reduction in juvenile survival and expected fecundity by C. capitata (Papaj et al., 1989). Despite this, however, C. capitata flies prefer to use existing punctures previously made by conspecific females (Papaj et al., 1989, 1992). The function of this behavior has been explained as an adaptive advantage for the fly when she has limited foraging time, and the benefit of using an existing oviposition puncture is larger than that of boring a new one in terms of the costs of time, predation, and larval competition (Papaj et al., 1992). Our study showed that at a relatively high host egg density per cluster, not all eggs in a cluster could be parasitized by F. arisanus. Thus, oviposition into an existing clutch by the host fly may confer an additional advantage: that of a reduced likelihood of parasitism or partial refuge within larger clutches of host eggs.

Fopius arisanus is currently the dominant parasitoid of fruit flies in Hawaii (Wong and Ramadan, 1984; Vargas et al., 1993; Purcell et al., 1998). The success of this parasitoid among many introduced species has been 
attributed to its competitive superiority against larval parasitoids (van den Bosch and Haramoto, 1953; Wang and Messing, 2002, 2003). Our study also suggests that effective searching behavior and high foraging efficiency may be additional factors leading to higher parasitism rates by $F$. arisanus. Host discrimination and a success-motivated search strategy ensure a thorough exploitation of host resources by this introduced biological control agent.

\section{ACKNOWLEDGMENTS}

We thank Terri Moats, Kauai Agricultural Research Center, University of Hawaii, for her assistance in this study and the USDA-ARS Pacific Basin Agricultural Research Center, Honolulu, for kindly providing parasitoids and host eggs. We also thank Dr. Eric Wajnberg for his valuable comments on an early draft of this paper. This research was supported by USDA-ARS Grant 5853208147 to R.H.M. This is paper No. 4656 in the UH College of Tropical Agriculture and Human Resources Journal Series.

\section{REFERENCES}

Bautista, R. C., Harris, E. J., and Lawrence, P. O. (1998). Biology and rearing of the fruit fly parasitoid Biosteres arisanus: Cues to insectary propagation. Entomol. Exp. Appl. 89: 7985 .

Bautista, R. C., Mochizuki, N., Spencer, J. R., Harris, E. J., and Ichimura, D. M. (1999). Massrearing of the tephritid fruit fly parasitoid Fopius arisanus (Hymenoptera: Braconidae). Biol. Control 15: 137-144.

Bess, H. A., van den Bosch, R., and Haramoto, F. H. (1961). Fruit fly parasites and their activities in Hawaii. Proc. Hawaii Entomol. Soc. 17: 367-578.

Charnov, E. L. (1976). Optimal foraging, the marginal value theorem. Theor. Popul. Biol. 9: 129-136.

Driessen, G., and Bernstein, C. (1999). Patch departure mechanisms and optimal host exploitation in an insect parasitoid. J. Anim. Ecol. 68: 445-459.

Driessen, G., Bernstein, C., van Alphen, J. J. M., and Kacelnik, A. (1995). A count-down mechanism for host search in the parasitoid Venturia canescens. J. Anim. Ecol. 64: 117-125.

Dukas, R., and Duan, J. J. (2000). Potential fitness consequence of associative learning in a parasitoid wasp. Behav. Ecol. 11: 536-543.

Field, S. A., and Keller, M. A. (1993). Courtship and intersexual signalling in the parasitic wasp Cotesia rubecula (Hymenoptera: Braconidae). J. Insect Behav. 6: 737-750.

Godfray, H. C. J. (1994). Parasitoids: Behavioural and Evolutionary Ecology, Princeton University Press, Princeton, NJ.

Goodman, L. A. (1968). The analysis of cross-classified data: independence, quasiindependence, and interactions in contingency tables with or without missing entries. $J$. Am. Stat. Assoc. 63: 1091-1131.

Greany, P. D., Tumlinson, J. H., Chambers, D. L., and Boush, M. G. (1977). Chemically mediated host finding by Biosteres (Opius) longicaudata, a parasitoid of tephritid fruit fly larvae. $J$. Chem. Ecol. 2: 189-195.

Haramoto, F. H. (1953). The Biology of Opius oophilus Fullaway (Braconidae: Hymenoptera), M.Sc. thesis, University of Hawaii, Honolulu. 
Haramoto, F. H., and Bess, H. A. (1969). Recent studies on the abundance of the Oriental and Mediterranean fruit flies and the status of their parasites. Proc. Hawaii Entomol. Soc. 20: 551-566.

Harris, E. J., and Bautista, R. C. (1996). Effects of fruit fly host, fruit species, and host egg to female parasitoid ratios on the laboratory rearing of Biosteres arisanus. Entomol. Exp. Appl. 79: 187-194.

Liquido, N. J. (1991). Effect of ripeness and location of papaya fruits on the parasitization rates of oriental fruit fly and melon fly (Diptera: Tephritidae) by braconid (Hymenoptera) parasitoids. Environ. Entomol. 20: 1732-1736.

Luck, R. F. (1990). Evaluation of natural enemies for biological control: a behavioral approach. Trends Ecol. Evol. 5: 196-199.

Messing, R. H., and Jang, E. B. (1992). Response of the fruit fly parasitoid Diachasmimorpha longicaudata (Hymenoptera: Braconidae) to host fruit stimuli. Environ. Entomol. 21:11891195.

Nelson, J. M., and Roitberg, B. D. (1995). Flexible patch time allocation by the leafminer parasitoid, Opius dimidiatus. Ecol. Entomol. 20: 245-252.

Papaj, D. R., Roitberg, B. D., and Opp, S. B. (1989). Serial effects of host infestation on egg allocation by the Mediterranean fruit fly: A rule of thumb and its functional significance. J. Anim. Ecol. 58: 955-970.

Papaj, D. R., Averill, A. L., Prokopy, R. J., and Wong, T. T. Y. (1992). Host-marking pheromone and use of previously established oviposition sites by the Mediterranean fruit fly. J. Insect Behav. 5: 583-598.

Purcell, M. F. (1998). Contribution of biological control to integrated pest management of tephritid fruit flies in the tropic and subtropics. Int. Pest Manage. Rev. 3: 63-83.

Purcell, M. F., Jackson, C. G., Long, J. P., and Batchelor, M. A. (1994). Influence of guava ripening on parasitism of the oriental fruit fly, Bactrocera dorsalis (Hendel) (Diptera: Tephritidae), by Diachasmimorpha longicaudata (Ashmead) (Hymenoptera: Braconidae) and other parasitoids. Biol. Control 14: 396-403.

Purcell, M. F., Herr, J. C., Messing, R. H., and Wong, T. T. Y. (1998). Interaction between augmentatively released Diachasmimorpha longicaudata (Hymenoptera: Braconidae) and a complex of opiine parasitoids in a commercial guava orchard. Biol. Sci. Tech. 8: 139151.

Quimio, G. M., and Walter, G. H. (2000). Swarming, delayed sexual maturation of males and mating behavior of Fopius arisanus (Sonan) (Hymenoptera: Braconidae). J. Insect Behav. 13: 797-813.

Ramadan, M. M., Wong, T. T. Y., and Beardsley, J. W. (1992). Reproductive behavior of Biosteres arisanus (Sonan) (Hymenoptera: Braconidae), an egg-larval parasitoid of the oriental fruit fly. Biol. Control 2: 28-34.

Ramadan, M. M., Wong, T. T. Y., and Mclnnis, O. O. (1994). Reproductive biology of Biosteres arisanus (Sonan), an egg-larval parasitoid of the oriental fruit fly. Biol. Control 4: 93-100.

Rice, W. R. (1989). Analyzing tables of statistical tests. Evolution 43: 223-225.

Rosenheim, J. A., and Mangel, M. (1994). Patch-leaving rules for parasitoids with imperfect host discrimination. Ecol. Entomol. 19: 374-380.

van Alphen, J. J. M., and Galis, F. (1983). Patch time allocation and parasitization efficiency of Asobora tabida Nees, a larval parasitoid of Drosophila. J. Anim. Ecol. 52: 937-952.

van Alphen, J. J. M., and Vet, L. E. M. (1986). An evolutionary approach to host finding and selection. In Waage, J. K., and Greathead, D. (eds.), Insect Parasitoids, Academic Press, London, pp. 23-61.

van den Bosch, R., and Haramoto, F. H. (1953). Competition among the parasites of the Oriental fruit fly. Proc. Hawaii Entomol. Soc. 15: 201-206.

van Lenteren, J. C. (1991). Encounters with parasitized hosts: To leave or not to leave a patch. Netherlands J. Zool. 41: 144-157.

Vargas, R. I., Stark, J. D., Prokopy, R. J., and Green, T. A. (1991). Response of oriental fruit fly (Diptera: Tephritidae) and associated parasitoids (Hymenoptera: Braconidae) to different color spheres. J. Econ. Entomol. 84: 1503-1507. 
Vargas, R. I., Stark, J. D., Uchida, G. K., and Purcell, M. (1993). Opiine parasitoids (Hymenoptera: Braconidae) of oriental fruit fly (Diptera: Tephritidae) on Kauai Island, Hawaii: Islandwide relative abundance and parasitism rates in wild and orchard guava. Environ. Entomol. 22: 246-253.

Vos, M., Hemerik, L., and Vet, L. E. M. (1998). Patch exploitation by the parasitoids Cotesia rubecula and Cotesia glomerata in multi-patch environments with different host distributions. J. Anim. Ecol. 67: 774-783.

Waage, J. K. (1979). Foraging for patchily distributed hosts by the parasitoid, Nemeritis canescens. J. Anim. Ecol. 48: 353-371.

Waage, J. K. (1983). Aggregation in field parasitoid populations: foraging time allocation by a population of Diadegma (Hymenoptera, Ichneumonidae). Ecol. Entomol. 8: 447-453.

Wang, X. G., and Keller, M. A. (2002). A comparison of the host-searching efficiency of two larval parasitoids of Plutella xylostella. Ecol. Entomol. 27: 105-114.

Wang, X. G., and Keller, M. A. (2003). Patch time allocation by the parasitoid Diadegma semiclausum (Hymenoptera: Ichneumonidae). I. Effect of inter-patch distance. J. Insect Behav. 16: 279-294.

Wang, X. G., and Messing, R. H. (2002). Newly imported larval parasitoid pose minor competitive risk to the extant egg-larval parasitoid of fruit flies in Hawaii. Bull. Entomol. Res. 92: 423-429.

Wang, X. G., and Messing, R. H. (2003). Intra- and interspecific competition by Fopius arisanus (Hymenoptera: Braconidae) and Diachasmimorpha tryoni (Hymenoptera: Braconidae), parasitoids of the Mediterranean fruit fly Ceratitis capitata (Diptera: Tephritidae). Biol. Control 27: 251-259.

Wharton, R. A. (1989). Classical biological control of fruit infesting Tephritidae. In Robinson, A., and Harper, G. (eds.), World Crop Pests, Fruit Flies: Their Biology, Natural Enemies, and Control, Vol. 3b, Elsevier Science, Amsterdam, pp. 303-313.

Wharton, R. A., and Gilstrap, F. E. (1983). Key to and status of opiine Braconid (Hymenoptera) parasitoids used in biological control of Ceratitis capitata and Dacus, s.l. (Diptera: Tephritidae). Ann. Entomol. Soc. Am. 76: 721-742.

Wong, T. T. Y., and Ramadan, M. M. (1987). Parasitization of the Mediterranean and Oriental fruit flies (Diptera: Tephritidae) in the Kula area of Maui, Hawaii. J. Econ. Entomol. 80: 77-80.

Wong, T. T. Y., Mochizuki, N., and Nishimoto, J. I. (1984). Seasonal abundance of parasitoids of the Mediterranean and Oriental fruit flies (Diptera: Tephritidae) in the Kula area of Maui, Hawaii. J. Econ. Entomol. 73: 140-145. 